\title{
Belphégor
}

\section{La méthode de recherche-création en Arts Plastiques :prescription, prospective et mise à l'épreuve de l'objet}

Anthony Rageul

\section{(2) OpenEdition}

Journals

Édition électronique

URL : https://journals.openedition.org/belphegor/3893

DOI : 10.4000/belphegor.3893

ISSN : 1499-7185

Éditeur

LPCM

Référence électronique

Anthony Rageul, «La méthode de recherche-création en Arts Plastiques :prescription, prospective et mise à l'épreuve de l'objet », Belphégor [En ligne], 19-1 | 2021, mis en ligne le 22 juin 2021, consulté le 08 juillet 2021. URL : http://journals.openedition.org/belphegor/3893 ; DOI : https://doi.org/10.4000/ belphegor.3893

Ce document a été généré automatiquement le 8 juillet 2021.

\section{(c) ()) (9)}

Belphégor est mis à disposition selon les termes de la Licence Creative Commons Attribution - Pas d'Utilisation Commerciale - Pas de Modification 4.0 International. 


\title{
La méthode de recherche-création en Arts Plastiques :prescription, prospective et mise à l'épreuve de l'objet
}

\author{
Anthony Rageul
}

La méthode de recherche-création propre aux arts plastiques place l'universitaire dans une position atypique: celle de chercheur-artiste, impliqué lui-même dans la construction de l'objet qu'il se propose d'étudier. Si on peut justifier cette méthode en faisant des analogies entre la démarche scientifique et le processus de création de l'artiste (Toulouse 2012), cela n'est pas sans poser problème en pratique. Ainsi, si les arts numériques et technologiques ont permis d'établir de nouvelles formes de coopération à travers des partenariats institutionnels associant création et ingénierie, la valorisation de ces travaux transdisciplinaires peut parfois poser des difficultés (Fourmentraux 2011). Ces coopérations courent par exemple le risque de voir la partie technologique devenir la vassale de la partie artistique, et inversement. La nécessité pour l'ingénierie de voir la recherche déboucher sur une application industrielle ou commerciale peut être loin des préoccupations de l'artiste, et réciproquement. Plus généralement, le problème de la recherche-création est amplement documenté par la littérature. Dans les écoles d'art, les accords de Bologne en 1999 ont aligné les cursus sur le système Licence-Master-Doctorat. C'est donc depuis ces accords que se cristallisent les débats sur la construction de troisièmes cycles et d'équipes de recherche (quelles que soient leurs formes et dénominations). À l'université, c'est la place à accorder à la pratique dans le cadre de la thèse qui est encore discutée aujourd'hui. Dans les deux cas, le jeune chercheur peut se voir soumis à des injonctions diverses, parfois contradictoires, tant sur la forme (avec ou sans écrit, d'un format classique ou plus souple) que sur la méthodologie (avec des injonctions à la scientificité qui peuvent être difficiles à conjuguer avec l'imprévisibilité du processus de création). En effet, on s'est d'abord demandé si la partie "création " produisait bien de la connaissance. Si une réponse positive semble faire consensus aujourd'hui, la façon dont 
la méthode de recherche-création produit cette connaissance et, en conséquence, la valeur et la pertinence de cette dernière, fait toujours l'objet de questions. L'ambition de ce texte est d'apporter une contribution à ces réflexions. On posera pour cela deux questions. Premièrement, comment la recherche-création produit-elle de la connaissance? Et deuxièmement, quels types de connaissance est-elle susceptible de produire?

2 Je partirai de ma propre expérience du doctorat en relevant brièvement les points saillants de la méthodologie de recherche-création appliquée à la bande dessinée numérique et en pointant les difficultés rencontrées. Puis je mènerai un travail de comparaison de ma thèse avec trois autres thèses portant sur le même objet - la bande dessinée numérique - mais qui relèvent d'autres champs disciplinaires et dont les méthodologies n'impliquent pas la création. Ces thèses possèdent en effet des points communs et des caractéristiques intéressantes, outre leur objet. D'une part, elles ont toutes été soutenues entre 2010 et 2016 , période particulière qui a vu une grande émulation des différents acteurs et observateurs de la bande dessinée numérique en France. Ces quatre thèses sont profondément ancrées dans ce contexte auquel elles réagissent chacune à leur manière. Il n'est d'ailleurs pas anodin qu'elles soient les premières à avoir été soutenues sur cet objet en France et qu'elles offrent à elles quatre une vue panoramique de la bande dessinée numérique et de ses grands enjeux durant cette période particulière. Ces points communs et cette complémentarité autorisent un travail de comparaison. D'autre part, elles abordent toutes la question de la création. En gardant à l'esprit qu'elles le font depuis leur point de vue respectif et pour répondre à des problématiques différentes, la comparaison de la façon dont chacune embrasse les questions de création de bande dessinée numérique permettra de mettre en évidence les apports spécifiques de la création comme méthode de recherche sur cet objet. Le cas échéant, certains de ces apports pourront être généralisables à la méthode de recherche-création indépendamment de l'objet ou du contexte considérés.

\section{La recherche-création au croisement d'un contexte et d'une pratique singulière}

3 Avant d'aller plus loin, il n'est pas inutile de rappeler le contexte particulier qui caractérise la période dans laquelle s'inscrivent les quatre thèses. Les années 2010 à 2016 ont marqué un tournant pour la bande dessinée numérique. Après plus de quinze années d'expérimentations formelles et plastiques, diverses initiatives éditoriales ont vu le jour de la part d'éditeurs, d'auteurs ou d'autres acteurs ${ }^{1}$. Les éditeurs traditionnels se concentraient sur l'adaptation de leur catalogue papier dans des formats numériques dits « homothétiques » souvent peu respectueux des œuvres originales. Des auteurs y opposaient une bande dessinée numérique dite «native ", conçue dès le départ pour les supports numériques ${ }^{2}$, mais qui restait malgré cela limitée à une poignée de formats, d'ailleurs devenus des standards depuis lors. Dans ce cadre, auteurs, éditeurs et chercheurs défendaient tour à tour des visions et définitions différentes de la bande dessinée numérique. Des divergences fortes s'exprimaient, marquées soit par un retranchement derrière les théories traditionnelles de la bande dessinée papier, soit par une volonté d'élargir ou de se dégager de ces théories ${ }^{3}$. Ces débats innervent les quatre thèses analysées ici. 
Ma pratique artistique et ma propre thèse s'inscrivent dans ce contexte propre à la bande dessinée numérique. Elles sont de surcroît tributaires du contexte de recherche que j'évoquais en introduction, à savoir celui d'une discipline dont la méthode principale est instable et conduit à des injonctions parfois contradictoires. Ainsi, comme nombre de thèse en Arts Plastiques, la mienne n'évite pas la discussion d'ordre épistémologique sur la recherche-création - ce dont j'aimerais témoigner ici. Dans son introduction, j'ai défini ma méthodologie comme "un inextricable dialogue dont émerge une pensée, qui s'incarne sous deux formes, l'une mentale, l'autre sensible. C'est une pensée qui s'actualise, dans un seul et même mouvement maïeutique, dans une production artistique et une production théorique. » (Rageul $2014: 19)$ Je concède volontiers aujourd'hui que cette formulation était trop emphatique. Cependant, elle traduit bien la difficulté qu'il y a à appréhender ce type de travaux: la création plastique est tout autant une trace et un produit de la recherche que l'est le traditionnel volume de cinq cents pages. De plus, la forme discursive ne prévaut pas sur la forme sensible; et cette dernière ne se contente pas d'illustrer le propos théorique. Les deux formes doivent se suffire à elles-mêmes. À cet égard, il n'est pas étonnant que j'aie - comme d'autres - réalisé des objets à mi-chemin entre œuvre et support pédagogique, telle que la conférence-performance Cache-cache en $2013^{4}$ - qui dévoile au public mon processus de création - ou la bande dessinée interactive Romuald et le tortionnaire en 2012 - dont la fin brise l'illusion de pouvoir conférée au lecteur par l'interactivité. Mais l'élément le plus important pour saisir l'importance de la pratique est peut-être le premier terme employé : celui de «dialogue ». En effet, c'est une véritable partie de ping-pong qui s'est jouée. Des avancées sur le plan théorique ont régulièrement remis en cause des propositions plastiques. Des propositions plastiques m'ont contraint à réinterroger certaines conclusions provisoires sur le plan théorique. Le moi-artiste et le moi-chercheur ont donc passé ces quatre années à se renvoyer la balle, se posant tour à tour et mutuellement des questions ou s'apportant des réponses. L'alternance libre des pronoms je et nous dans ma thèse témoigne de ce dialogue. L'usage de la première personne a souvent révélé la parole "polémique » ou «militante » de l'artiste, que le chercheur a dû apprendre à réfréner avec l'aide de ses directeurs de thèse. Cette parole est la manifestation d'une réaction - parfois épidermique : celle de l'artiste face au contexte dans lequel se trouve alors la bande dessinée numérique sur le plan créatif comme sur le plan théorique.

\section{Présentation des quatre thèses}

5 Soutenue en 2010 à l'Université Paris 8 et dirigée par Daniel Danétis, la thèse en Esthétique, Sciences et Technologies des Arts de Magali Boudissa s'intitule La Bande dessinée entre la page et l'écran : étude critique des enjeux théoriques liés au renouvellement du langage bédéique sous influence numérique. Boudissa y confronte la bande dessinée à son changement de support, du papier au numérique. Elle fait l'hypothèse que le "système" de la bande dessinée est bousculé par le numérique à travers deux problématiques : celle de l'espace de l'écran et celle, bicéphale, du multimédia et de l'interactivité. L'approche adoptée relève de la sémiotique des médias. Boudissa fait le choix d'arrêter une définition précise - bien que large - de la bande dessinée, issue des travaux de Thierry Groensteen (1999). Elle y confronte un corpus d'œuvres et expérimentations de la fin des années 90 et du début des années 2000. Les points de 
continuité et de ruptures ainsi constatés la conduisent à juger de la conformité ou nonconformité des exemples qu'elle présente avec le cadre théorique traditionnel. Les différences mises en évidence sont le plus souvent considérées en termes de "perte » ou de « gain » pour la bande dessinée.

Julien Falgas a soutenu sa thèse en Sciences de l'Information et de la Communication en 2014. Elle s'intitule Raconter à l'ère numérique: auteurs et lecteurs héritiers de la bande dessinée face aux nouveaux dispositifs de publication et a été dirigée par Brigitte Simonnot à l'Université de Lorraine. Falgas réfute l'idée d'une définition et d'une ontologie de la bande dessinée numérique. Il voit plutôt le rattachement de récits numériques émergents à la bande dessinée comme une manifestation du phénomène de convergence des médias à l'ère numérique. L'objet d'étude de Falgas n'est donc pas les " contenus narratifs» mais les "usages des auteurs et des publics qui lisent ou racontent des histoires sur supports numériques en se réclamant de l'héritage de la bande dessinée.» (Falgas 2014: 26) Il formule l'hypothèse que "la recherche des marques d'indexicalité [chez les auteurs et lecteurs] doit [lui] permettre de mettre en évidence les négociations qui aboutissent à une stabilisation des usages » (Falgas 2014 : 32). Cet objet lui donne aussi l'occasion d'observer un phénomène de convergence des médias en train de se faire, et non après-coup. Se revendiquant de l'ethnométhodologie, il conduit des questionnaires en ligne et des séries d'entretiens auprès des auteurs et des lecteurs des récits numériques Les Autres Gens et Média Entity. Parmi d'autres observations, ces deux études de cas mettent en avant que l'émergence d'une bande dessinée contemporaine sur support numérique fait apparaître une référence constante aux cadre éditoriaux et esthétiques de la bande dessinée, d'une part, et de l'audiovisuel, d'autre part. Elles font voir également que les références à d'autres cadres - tels que celui des plateformes contributives - de la part des auteurs n'ont pas ou peu été partagées par les lecteurs, ce qui est particulièrement flagrant dans le cas de Media Entity.

7 J'ai quant à moi soutenu un peu plus d'un mois après Falgas une thèse en Arts Plastiques à l'Université Rennes 2 intitulée $L a$ Bande dessinée saisie par le numérique: formes et enjeux du récit reconfiguré par l'interactivité. Elle a été dirigée par Ivan Toulouse, alors enseignant-chercheur-artiste à l'Université Rennes 2 et Benoît Berthou, enseignant-chercheur en Sciences de l'Information et de la Communication à Paris 13. Constatant que les théories traditionnelles de la bande dessinée sont insuffisantes pour prendre en charge mes propres créations ainsi que la partie la plus expérimentale de la bande dessinée numérique, je postule que la bande dessinée numérique est un nouveau médium (au sens artistique) et un nouveau média (au sens d'objet de communication et de fait culturel) ; c'est-à-dire un moyen d'expression artistique dont le périmètre et la dénomination sont globalement et implicitement reconnus et partagés par ses différents acteurs (artistes, spectateurs, institutions, observateurs). Ce postulat permet de sortir de la comparaison entre bande dessinée et bande dessinée numérique, ce à quoi se refusent par exemple Magali Boudissa ou le théoricien Thierry Groensteen (2011). Désormais autonome, la bande dessinée numérique peut alors être étudiée pour elle-même et définie à l'aide de critères adaptés. La problématique reprend le leitmotiv de ma pratique artistique: qu'est-ce qu'une bande dessinée numérique qui exploite véritablement les propriétés et le potentiel plastique et narratif des technologies pour produire du sens? Mes recherches théoriques, mes créations - telles que celles citées plus haut - et activités annexes ${ }^{5}$ me conduisent donc à tenter de cerner pour la 
première fois la bande dessinée numérique dans ses spécificités, et non sous le strict angle d'une confrontation avec la bande dessinée traditionnelle. J'essaie de la doter d'un cadre théorique propre, qui s'appuie pour partie sur une réactualisation des théories traditionnelles de la bande dessinée, et qui emprunte surtout beaucoup aux théories du jeu et du jeu vidéo, aux théories des arts et littératures numériques, à la sémiotique des médias numériques et à la narratologie ${ }^{6}$. Au terme de mes travaux, je propose cinq notions clés qui permettent d'appréhender plus finement ce média: gameplay, narrateur-arbitre, modularité, poésie et, la plus importante, récit-interface. Cette dernière notion traduit la place centrale de l'interface dans les processus d'écriture et de "lectacture $»^{7}$ du récit de bande dessinée numérique ${ }^{8}$.

Enfin, Philippe Paolucci soutient en 2016 une thèse en Sciences de l'Information et de la Communication sous le titre La Bande dessinée numérique : entre rupture et continuité. Pour une approche socio-sémiotique de la transition numérique du neuvième art: l'exemple de la revue en ligne Professeur Cyclope, réalisée à l'Université Lyon 2 Lumière sous la direction de Pascal Robert et Patrick Mpondo-Dicka. Paolucci rappelle que si tous les médias numériques sont codés en un unique matériau, ils conservent les propriétés sémiotiques de traditions culturelles. Dès lors, il se demande comment les auteurs s'approprient la tension entre héritage du neuvième art et nouvelles expériences numériques. Il souhaite également étayer l'idée même de traiter de la bande dessinée numérique au sein des Sciences de l'Information et de la Communication au moyen d'une démarche socio-sémiotique. Il justifie donc longuement les outils théoriques mis en œuvre, entre autres la notion d'écart. C'est à travers cette notion qu'il conduit une étude fouillée d'un corpus de bandes dessinées numériques parues dans la revue numérique Professeur Cyclope qu'il complète par des entretiens avec leurs auteurs. Il identifie deux grandes tendances: d'une part une forte linéarisation des récits numériques, d'autre part une tendance à l'hybridation où la logique de l'écart est cruciale pour des auteurs cherchant un équilibre entre proximité et éloignement vis-àvis des formes traditionnelles de la bande dessinée. Parmi d'autres points, ces travaux mènent Paolucci à insister sur la nécessité de construire une rhétorique du texte numérique.

9 Ces thèses se montrent très complémentaires, abordant la bande dessinée numérique tour à tour comme un médium artistique, un média, un objet culturel et un phénomène communicationnel. Elles s'intéressent soit aux propriétés intrinsèques de l'objet, soit au halo des acteurs qui gravitent autour. Elles brassent donc différents aspects du même objet et offrent toutes ensemble une vision panoptique et transdisciplinaire de la bande dessinée numérique dans une période donnée.

\section{Différentes approches de la question de la création}

Afin de comparer la manière dont chaque thèse produit des connaissances en utilisant la création ou en l'examinant, j'ai procédé à un relevé de tous les passages qui s'inscrivaient dans une telle approche. Ce travail renseigne sur les diverses thématiques qui ont intéressé les chercheurs sous cette question de la création ${ }^{9}$. Dès lors, il devient aisé de mettre en évidence quelles thématiques apparaissent dans ma propre thèse et ne se trouvent pas dans les autres, ou s'y trouvent dans une moindre mesure, et d'identifier ainsi ce qui fait sa singularité et en quoi la méthodologie de la recherchecréation appliquée à la bande dessinée numérique complète les méthodologies des 
autres champs disciplinaires. Il m'a paru nécessaire de procéder de cette manière méthodique, y compris pour ma propre thèse, de manière à garantir sinon une meilleure objectivité face à ces textes, au moins un recul suffisant. D'ailleurs, cette précaution s'est trouvée tout à fait justifiée quand les informations apportées par ce relevé ont fait voler en éclat certains préjugés que je pouvais avoir. Je m'attendais notamment à ce que Falgas, Boudissa et Paolucci abordent les questions techniques de manière moins précise que moi, dans la mesure où j'entretiens par le biais de la création une grande proximité avec la technique. Or, il n'en est rien et seule cette lecture méthodique pouvait me prémunir contre ces mauvaises intuitions et ces a priori problématiques.

11 Cela étant précisé, les passages relevés peuvent être classés par thématique. Certaines sont communes à toutes les thèses tandis que d'autres n'apparaissent que dans une ou deux, ce qui laisse penser qu'elles caractérisent la discipline et/ou l'approche particulière du chercheur et, le cas échéant, l'approche par la recherche-création. Les trois thématiques communes sont les suivantes: les aspects concrets et matériels du processus de création (ce qui comprend les contraintes et difficultés techniques et le contexte dans lequel est produite l'œuvre), la poïétique (c'est-à-dire des réflexions d'ordre général sur le processus de création non appliquées à un cas particulier), la recherche des intentions (c'est-à-dire l'explicitation des choix techniques et artistiques faits par les artistes). Ces trois thématiques reflètent une volonté commune de parvenir à identifier et nommer les non-dits de la création en train de se faire. D'autres thématiques n'apparaissent pas partout. Falgas et Paolucci s'intéressent par exemple à la façon dont les artistes eux-mêmes, à partir de leur propre expérience de la création, définissent la bande dessinée numérique. On peut souligner aussi que, parmi les aspects techniques de la création, Falgas pointe en particulier l'émergence de nouvelles pratiques liées aux nouveaux outils de communication. Deux autres thématiques semblent liées à l'ancrage disciplinaire en art car elles n'apparaissent singulièrement que dans les travaux de Boudissa et les miens. La première réside dans une approche de la création que je qualifierais de prospective; la seconde dans une démarche prescriptive. Pour terminer, le relevé met en évidence une dernière thématique, présente seulement dans ma thèse : l'utilisation de mon processus de création comme matrice d'une méthodologie de recherche théorique et d'une rhétorique.

\section{Analyse comparative}

Ce sont les différences entre les approches de chacun qui m'intéressent. Je vais donc me pencher plus particulièrement sur les thématiques qui ressortent et sur celles qui, bien que communes aux quatre thèses, sont traitées de manières différentes.

13 La première remarque concerne justement une thématique commune. Les quatre thèses s'attachent à rechercher quelles sont les intentions des artistes. Cependant, une différence majeure apparaît quand on s'intéresse aux méthodologies employées. Cette différence marque une césure entre les travaux de Falgas et Paolucci d'une part, et ceux de Boudissa et moi d'autre part. En effet, les deux premiers travaillent sur des témoignages issus d'entretiens menés auprès des artistes. Les informations obtenues sont de première main et correspondent aux intentions exprimées par les artistes euxmêmes. À l'inverse, Boudissa ne fait pas mention d'entretiens avec des artistes; tout au plus cite-t-elle de temps en temps des propos d'artistes extraits d'interviews qu'ils ont 
données ici ou là. De même, je ne m'appuie sur les propos des artistes qu'à deux reprises et au sujet de cas bien précis. Mais pour l'essentiel, Boudissa et moi déduisons les intentions des artistes de l'analyse esthétique et sémiotique que nous faisons de leurs œuvres. En ce sens, les intentions que nous leur prêtons ne peuvent être que des suppositions. Pour preuve : nous leur prêtons toujours des intentions claires alors que les propos relayés par Falgas et Paolucci manifestent parfois le flou dans lequel se trouvent les artistes quand il s'agit de préciser leurs intentions. Falgas et Paolucci adoptent une approche sociologique ${ }^{10}$ alors que Boudissa et moi nous en tenons exclusivement aux œuvres.

14 La thématique suivante voit la création abordée d'une manière que j'ai qualifiée de prospective. Elle n'apparait que dans la thèse de Boudissa et la mienne. Elle consiste dans le fait d'envisager et de décrire des potentialités formelles et narratives que le numérique peut offrir à la bande dessinée mais que peu d'auteurs à notre connaissance ont mises en pratique au moment où l'on parle ${ }^{11}$. Cela s'incarne par des phrases au conditionnel, voire au futur. Un exemple parmi beaucoup d'autres chez Boudissa :

Le zoom pourrait ainsi être utilisé comme un nouvel outil narratif dans la palette du récit bédéique [...] L'auteur attirerait l'attention du lecteur sur des éléments particuliers du récit en jouant sur leur lisibilité : pourquoi ne pas imaginer d'insérer une toute petite vignette au milieu d'autres vignettes de taille «normale " sur laquelle le lecteur devrait zoomer plusieurs fois pour la lire? (Boudissa 2009 : 92)

En somme, et pour reprendre une expression utilisée par Paolucci pour qualifier cette approche de la création, nous faisons référence à des «formes possiblement actualisables $»^{12}$ (Paolucci 2016: 267) que nous intégrons à notre réflexion comme si elles existaient. Ce type de proposition trouve un illustre précédent dans Réinventer la bande dessinée de Scott McCloud (2001). En ce qui concerne mes travaux, ces propositions sont des possibles que je laisse à disposition, espérant que d'autres artistes s'en emparent ${ }^{13}$.

16 À travers les suggestions que je formule, l'approche prospective de la création devient une démarche prescriptive. Boudissa partage également cette démarche, mais elle se manifeste différemment chez elle, non plus à travers la suggestion, mais à travers de très nombreuses recommandations qu'on devine destinées aux artistes ${ }^{14}$. Il s'agit de s'assurer de produire des interfaces qui soient parfaitement ergonomiques, mais aussi de ne pas dénaturer la bande dessinée à travers des formes trop éloignées du cadre définitionnel traditionnel. Ces recommandations reposent sur les jugements émis par Boudissa en ce qui concerne les gains et pertes de la bande dessinée dus au numérique. Nos deux démarches, qui n'apparaissent pas chez Falgas et très sporadiquement chez Paolucci, traduisent notre inscription dans une position de créateur ou, plus précisément, d'inventeur - même si Boudissa n'est pas praticienne. Elles complètent évidemment des démarches portant exclusivement sur l'existant dans la mesure où elles ouvrent l'objet sur de nouvelles perspectives, fussent-elles de pures potentialités. Elles posent alors un cadre qui permet d'étendre la réflexion théorique. À la question " que se passe-t-il quand... ? » on ajoute « et que se passerait-il si...? ».

De façon plus générale, le processus de création peut servir de matrice au déploiement d'une méthodologie de recherche et à une rhétorique pour la transcrire. Un des six chapitres de ma thèse repose sur une telle référence au processus de création. Il s'agit du chapitre que je consacre à l'analyse de l'interactivité telle qu'elle est mise en place dans le récit dessiné numérique. Cette présence de l'interactivité pose la question de l'interface et de la nature de l'image numérique. Afin d'en mener l'analyse, il fallait que 
je parvienne à délier ces trois niveaux inextricables. J'en suis venu à les aborder sous l'angle de l'écriture. Comment écrit-on une interface? Comment écrit-on l'interactivité ? Comment écrit-on une image numérique? Avant d'être formalisé, ce choix d'angle d'attaque était surtout une intuition, une évidence. Et quelle pouvait être l'origine de cette intuition sinon mon propre processus de création, qui implique en effet une écriture du récit sur les trois niveaux de l'interactivité, de l'interface et de l'image? Au niveau méthodologique, ces trois niveaux m'ont fourni une grille de lecture du problème que je voulais étudier. Au niveau rhétorique, j'ai rédigé ce chapitre en trois parties suivant ces trois niveaux. Pour éviter toute confusion, il faut bien voir que ma pratique et mon processus de création ne sont pas le cœur du propos du chapitre concerné. Ce dernier est consacré à un ensemble de questions théoriques bien plus larges. Il adapte à la bande dessinée numérique les principales typologies de l'interface, de l'interactivité et de l'image numérique établies par les théoriciens des arts numériques ${ }^{15}$. La méthode employée a ainsi permis un apport de connaissances générales en plus d'une meilleure compréhension de mon processus de création ${ }^{16}$. Il faut donc bien voir que ce qui est central ici, c'est le fait que la méthodologie de recherche et la rhétorique sont calquées sur mon processus de création indépendamment du fait de parler ou non de ce processus, et conduisent bien à une production de connaissances. C'est dans ce sens que je parle du processus de création et de la pratique comme matrices de la méthodologie et de la rhétorique.

\section{La création pour bousculer l'objet}

18 En jouant ce rôle de matrice, le processus de création et la pratique ne sont plus seulement un contenu qui alimente la réflexion du chercheur - qu'il soit lui-même praticien ou non. Ils deviennent un outil méthodologique à part entière. En l'occurrence, ils prolongent concrètement les démarches prospectives et prescriptives mentionnées plus haut. D'ailleurs, le plus souvent, j'actualise moi-même les formes possibles que j'imagine, c'est-à-dire que je les mets en application dans mes propres créations. Cela peut prêter le flanc à la suspicion, notamment parce que cela peut laisser entendre que j'aurais créé des objets ad hoc pour confirmer certaines hypothèses; ce qui relèverait d'une forme de biais de confirmation. Le trouble est d'autant plus difficile à dissiper qu'une grande partie des hypothèses que je formule sont à la fois et indistinctement des hypothèses théoriques et artistiques. Exemple : je me dis qu'il doit être possible de m'emparer de la souris pour en faire un élément de la narration à part entière - c'est mon hypothèse. Alors j'essaie, j'expérimente, notamment à travers Cache-cache, dans laquelle la souris devient un personnage de l'histoire. L'expérience valide ici mon hypothèse, aussi bien sur le plan artistique que théorique. Il est tentant de dresser un parallèle avec l'expérience de laboratoire des sciences dures, à l'instar de ce que fait Pierre Schaeffer. Ce dernier invente la notion de "production expérimentale " pour désigner les expérimentations radiophoniques qui alimentent ses recherches théoriques. "Pour lui, production et passage à l'antenne sont des actes analogues à des actes scientifiques, des hypothèses vérifiées ou non par la faisabilité de la production et par l'intérêt du public. » (Perriault 2012) Dans ce cadre, mes scrupules quant à un éventuel biais ne seraient pas fondés: dès lors que l'expérience fonctionne, l'hypothèse est validée. Ces scrupules ne seraient peut-être bien qu'un reliquat des injonctions contradictoires évoquées en introduction et auxquelles peut se voir confronté le doctorant ou le jeune chercheur. Quoi qu'il en soit, 
même si je trouve exagérée une telle analogie avec l'expérience de laboratoire, la méthodologie que j'applique relève d'une recherche fondamentale en art dont j'aimerais voir un jour des mises en application par d'autres artistes.

La recherche-création peine toujours à faire reconnaître et accepter ses méthodes et ses résultats, et ce d'autant plus qu'elle revendique son inscription dans une démarche de recherche fondamentale. C'est particulièrement problématique quand il s'agit de trouver le soutien des différentes institutions culturelles et du monde de la recherche ${ }^{17}$. Une dernière anecdote personnelle permettra d'illustrer les incompréhensions dont elle peut faire l'objet. Cette anecdote nous ramènera aux quelques mois qui ont précédé mon inscription en doctorat et, symboliquement, au début de ce texte, renvoyant aux difficultés notées par Fourmentraux. Durant l'année 2010, il a été question d'établir une convention $\mathrm{CIFRE}^{18}$ avec une société éditrice de jeux vidéo désirant se tourner vers la bande dessinée numérique pour diversifier ses activités. Les échanges - restés informels - auront été de très courte durée : dès lors qu'il s'est agi d'aborder le fond de mon projet de recherche, ce fut une fin de non-recevoir. Comment aurait-il pu en être autrement alors que mon travail consistait précisément à remettre en cause en grande partie le modèle créatif, éditorial et commercial de ce type de société ? Les questions que je soulevais dans le cadre d'une recherche fondamentale n'étaient pas compatibles avec les codes et les besoins d'une recherche appliquée visant un débouché commercial immédiat.

\section{Conclusion}

La recherche-création dans le corpus délimité pour ce texte possède plusieurs particularités. Outre les démarches prospectives et prescriptives, partagées également par des thèses ne reposant pas sur la création, elle fait aussi de la pratique une matrice de la méthodologie et de la rédaction de la thèse. Mais sa véritable spécificité semble être sa nature de recherche fondamentale et expérimentale. Son apport principal réside dans le rôle qu'y joue la création: celui d'expérience de laboratoire visant à démontrer ou réfuter une hypothèse théorique et plastique. Toutefois, le rôle méthodologique de la pratique ne s'y limite pas à l'expérience de laboratoire. Je lui donne un rôle bien plus profond et central sur lequel je fais tout reposer. Les prémisses et la problématique même de ma thèse sont une traduction directe de mes revendications artistiques, exprimant une défiance vis-à-vis des formats standards émergents de la bande dessinée numérique. La relecture méthodique à laquelle j'ai procédé montre d'ailleurs très clairement que plus j'avance dans mes travaux, affinant mon analyse et ma compréhension de la bande dessinée numérique en général et de ses formes les plus marginales, plus mes positionnements artistiques se renforcent en réaction au contexte dans lequel ma pratique prend place. En particulier, ma pratique s'oppose de plus en plus vivement aux formats standards et ma réflexion théorique finit par refuser de cantonner la bande dessinée numérique à un mode de réception exclusif qui serait la lecture, au détriment du visionnage et de l'interactivité. À ce stade, l'objet que j'étudie n'est plus seulement la bande dessinée numérique mais aussi le contexte dans lequel se place ma pratique et auquel elle réagit. De la sorte, la méthode de recherche-création consiste à utiliser ma pratique pour bousculer ce contexte et ainsi le questionner et éprouver ses limites. Cela n'apparaît pas chez Falgas et Paolucci qui s'en tiennent à l'observation de faits et de situations existantes. On en voit des 
frémissements chez Boudissa à travers sa démarche prospective et le fait qu'elle aussi affirme des positions esthétiques fortes à travers ses prescriptions. Toutefois, elle ne franchit jamais la frontière de la création en mettant en pratique elle-même ses propres propositions. De cette dernière différence entre les quatre thèses, on peut conclure que l'apport et le cœur de la méthode de recherche-création tiennent dans la formule suivante : provoquer délibérément des situations inédites pour mettre à l'épreuve son objet.

\section{BIBLIOGRAPHIE}

Bouchardon Serge (dir.), Un laboratoire de littératures. Littérature numérique et Internet., Paris, Bibliothèque publique d'information Centre Georges Pompidou, 2007.

Boudissa Magali, La Bande dessinée entre la page et l'écran :étude des enjeux théoriques liés au renouvellement du langage bédéique sous influence numérique, thèse non publiée, Paris 8, Paris, 2010.

Chomarat-Ruiz Catherine (dir.), De l'atelier au labo : inventer la recherche en art et design, Paris, Hermann, 2018.

Falgas Julien, « Thierry Groensteen, Bande dessinée et narration. Système de la bande dessinée 2 », Questions de communication, 31 décembre 2012, nº 22, p. 324-326.

Falgas Julien, Raconter à l'ère numérique : auteurs et lecteurs héritiers de la bande dessinée face aux nouveaux dispositifs de publication, thèse non publiée, Université de Lorraine, Metz, 2014. http:// www.theses.fr/2014LORR0112 [consulté le 30/10/2018]

Fourmentraux Jean-Paul, Art et science, Paris, CNRS Éditions, « Les essentiels d'Hermès », 2012.

Fourmentraux Jean-Paul (dir.), Artistes de laboratoire : recherche et création à l'ère numérique, Paris, Hermann, 2011.

Groensteen Thierry, Bande dessinée et narration. Système de la bande dessinée 2., Paris, Presses Universitaires de France, 2011.

McCloud Scott, Réinventer la bande dessinée, traduit par Jean-Paul Jennequin, Paris, Vertige graphic, 2002.

Paolucci Philippe, La Bande dessinée numérique : entre rupture et continuité. Pour une approche sociosémiotique de la transition numérique du neuvième art : l'exemple de la revue en ligne Professeur Cyclope., thèse non publiée, Université Lumière Lyon 2, Lyon, 2016.

Perriault Jacques, « Art, technique et mouvement social dans la genèse des théories sur la communication », dans Jean-Paul Fourmentraux (dir.), Art et science, Paris, CNRS Éditions, « Les essentiels d'Hermès », 2012, p. 163-183.

Rageul Anthony (alias Tony), « Daniel Merlin Goodbrey (entretien) », Du9, 2017 https:// www.du9.org/entretien/daniel-merlin-goodbrey/ [consulté le 11/11/2020].

Rageul Anthony, La Bande dessinée saisie par le numérique : formes et enjeux du récit configuré par l'interactivité, thèse non publiée, Université Rennes 2, 2014. http://www.theses.fr/2014REN20024 [consulté le 30/10/2018] 
Rageul Anthony, « Récit-interface. Une catégorie pour penser les récits numériques. », Ligeia, 2020, no 181-184, p. 122-131.

Réol Jean-Marc et Renucci Franck (dirs.), Hermès, la revue : L'artiste, un chercheur pas comme les autres, 72, 2, CNRS Éditions, 2015.

Sicard Monique (dir.), Chercheurs ou artistes? Entre art et science, ils rêvent le monde, Paris, Autrement, 1995.

Toulouse Ivan, Clair-obscur : essai sur la pensée créatrice, Paris, l'Harmattan, « Eurêka \& Cie », 2012.

Weissberg Jean-Louis, Présences à distance, Paris, Montréal, L’Harmattan, 1999.

\section{NOTES}

1. Notamment des fournisseurs de solutions logicielles pour la diffusion de contenus en ligne.

2. Leurs initiatives ne sont pas sans rappeler l'explosion de l'édition alternative dans les années 90. Il est d'ailleurs ironique de noter qu'un certain nombre d'éditeurs et d'auteurs alternatifs, attachés à l'objet-livre, refusent le numérique sans voir la proximité des problématiques rencontrées par la bande dessinée numérique avec celles rencontrés par la bande dessinée papier dans les années 90, notamment en ce qui concerne la nécessité de s'emparer des supports et de remettre en cause les modèles éditoriaux. Exceptions notables : la maison d'édition L'employé du moi et sa plateforme grandpapier.org ou plus récemment Hécatombe avec sa succursale Collection $R V B$ consacrée à la bande dessinée numérique.

3. La lecture critique que Julien Falgas (2012) fait de l'ouvrage Bande dessinée et narration de Thierry Groensteen (2011) fournit une bonne indication sur la teneur de ces débats.

4. Toutes mes créations sont accessibles et/ou documentées sur www.anthonyrageul.net.

5. Par exemple la conception et l'animation d'un atelier destiné à des auteurs invités en résidence par l'association Chifoumi en 2011. Cette activité a été une occasion de mettre entre parenthèses ma propre création pour étudier la création chez d'autres artistes à travers une expérience, au sens scientifique. J'ai proposé des outils et contraintes de création - au sens de l'Oulipo ou de l'Oubapo - afin de leur faire expérimenter la création de bande dessinée numérique et étudier la façon dont ils allaient l'aborder. Plus d'informations sur cette page: https:// www.pierrefeuilleciseaux.com/le-versant-numerique-durant-pfc3/. L'ensemble est bien sûr analysé longuement dans ma thèse.

6. La co-direction transdisciplinaire de la thèse témoigne d'ailleurs de cette nécessité d'emprunter des outils théoriques à diverses disciplines pour renouveler le cadre théorique traditionnel.

7. Emprunt à Jean-Louis Weissberg. À ma connaissance, la première occurrence apparait dans Weissberg, 1999.

8. Pour plus de développements, voir Rageul, 2020.

9. Ce travail renseigne également sur la fréquence de ces passages et donc sur la place accordée à la question de la création dans chacun de ces travaux. Toutefois, il ne s'agit que de grandes indications dans la mesure où il a très souvent été difficile de déterminer les bornes délimitant tel ou tel passage.

10. Partielle chez Paolucci, qui se revendique d'une approche socio-sémiotique dont la part sociologique intervient en second lieu.

11. À l'exception notable de pionniers tels que Daniel Merlin Goodrey. Plus d'information sur sa carrière dans l'entretien que j'ai mené (Rageul 2017).

12. Le passage dont est extraite cette expression est surprenant: "Boudissa et Rageul interrogent les propriétés sémio-esthétiques des formes actuellement en usage. Telle sera 
également notre posture. À aucun moment nous ne ferons référence à des formes possiblement actualisables. Plutôt qu'une sorte de foi en l'avenir, notre ambition est de décrire à l'échelle d'un corpus un existant aux contours flous. » Paolucci affirme donc que Boudissa et moi-même nous en tenons à des formes en usage, alors que nos textes sont truffés de propositions prospectives. Est-ce à mettre au compte d'une maladresse dans la formulation de Paolucci, d'une véritable incompréhension ou de propositions insuffisamment appuyées chez Boudissa et moi-même?

13. C'est déjà le cas avec ma proposition de planches de bandes dessinées dites " évolutives ", que j'ai expérimentées avec La justice est bovine et dont Emmanuel Espinasse et Johanna Schipper ont offert une magnifique mise en application avec Au paradigme (bande dessinée numérique dont la version bêta a été montrée lors de l'exposition «In Wonder » du 14 octobre au 31 décembre 2017 à la CIBDI d'Angoulême). Bien sûr, je ne prétends pas qu'ils se sont explicitement inspirés de ma proposition théorique!

14. Elles ne sont pas adressées explicitement aux artistes mais à qui d'autre pourraient-elles être destinées?

15. Cette révision des typologies repose en grande partie sur une prise en compte des compromis faits par les artistes avec ces dispositifs techniques.

16. Les trois niveaux d'écriture étaient un impensé de mon processus de création jusqu'à ce que je prenne conscience que le plan de ce chapitre de la thèse était justement calqué sur mon processus de création!

17. Le colloque « Réalités de la recherche (collective) en arts » organisé par l'unité de recherche CLARE de l'Université Bordeaux Montaigne en 2018 a justement été motivé par la nécessité d'expliquer aux partenaires institutionnels ce qu'était la recherche en art: ses méthodes, ses apports, ses spécificités.

18. La Convention Industrielle de Formation par la Recherche établit un partenariat entre un laboratoire, un doctorant et une entreprise. Elle facilite le recrutement du doctorant par une entreprise qui lui confiera un projet de recherche objet de sa thèse.

\section{RÉSUMÉS}

La méthode de recherche-création propre aux arts plastiques place l'universitaire dans une position atypique : celle de chercheur-artiste, impliqué lui-même dans la construction de l'objet qu'il se propose d'étudier. Si on peut justifier cette méthode en faisant des analogies entre la démarche scientifique et le processus de création de l'artiste (Toulouse 2012), cela n'est pas sans poser problème en pratique. Ainsi, si les arts numériques et technologiques notamment ont permis d'établir des partenariats institutionnels associant création et ingénierie, il apparaît que la partie création reste souvent la plus difficile à valoriser (Fourmentraux 2011). Je discute ici des apports et des écueils ou difficultés de cette méthode dans ma propre thèse portant sur la bande dessinée numérique (2014). Pour cela, je la mets en perspective avec trois thèses qui ont été soutenues sur cet objet entre 2010 et 2016 dans différents champs disciplinaires. Cette étude comparative met en lumière trois démarches plus spécifiques à la recherche-création: prescription, prospective et mise à l'épreuve de l'objet. 
INDEX

Mots-clés : recherche-création, bande dessinée numérique, épistémologie, art

\section{AUTEUR}

\section{ANTHONY RAGEUL}

Docteur en Arts Plastiques, Anthony Rageul est actuellement enseignantchercheur (ATER) en Arts Plastiques à I'Université Toulouse Jean Jaurès et membre du LARA-SEPPIA. A la fois théoricien et praticien, il est également auteur de bande dessinée papier et numérique, actif en ligne et dans le fanzinat. 